\title{
A A Case of Pterygopalatine Fossa Case \\ Report Arteriovenous Malformation Treated by Transarterial Embolization Using a Dual-Lumen Balloon Catheter
}

\author{
Sadayoshi Nakayama, ${ }^{1}$ Tomoaki Terada, ${ }^{1}$ Akira Wada, ${ }^{2}$ and Hajime Yabuzaki ${ }^{1}$
}

Objective: We report a rare case of pterygopalatine fossa arterial venous shunt disease with venous congestion of the superior ophthalmic vein (SOV) that was treated by transarterial balloon-assisted embolization.

Case Presentation: A 57-year-old man presented with congestion of the right bulbar conjunctiva, protrusion, and swelling of the right eyelid was admitted to our hospital. Angiography demonstrated an arteriovenous fistula (AVF) forming small congregated vessels in the pterygopalatine fossa fed by the branch of the ophthalmic artery (OA) and artery of the superior orbital fissure, draining into the SOV via the inferior ophthalmic vein (IOV). From the artery of the superior orbital fissure, transarterial embolization (TAE) with ONYX using a dual-lumen balloon catheter was performed. The patient was treated without complications.

Conclusion: TAE using transarterial balloon-assisted embolization with ONYX is effective for periorbital arteriovenous shunts, although special care is necessary to prevent the migration of ONYX into the OA.

Keywords > pterygopalatine fossa, dural arteriovenous fistula, arteriovenous malformation, transarterial embolization

\section{Introduction}

Concerning treatment for arteriovenous shunt disease of the head and neck, the development and introduction of non-adhesive liquid embolic substances, such as ONYX (Medtronic, Minneapolis, MN, USA) and PHIL (MicroVention, Tustin, CA, USA), has improved the results of endovascular treatment by transarterial embolization (TAE). ${ }^{1,2)}$ When performing TAE with liquid embolic substances in patients with arteriovenous shunt disease of the head and neck, the migration of liquid embolic substances into the ophthalmic artery (OA) or a feeder to the cranial

${ }^{1}$ Department of Neurosurgery, Showa University Fujigaoka Hospital, Yokohama, Kanagawa, Japan

${ }^{2}$ Department of Neurosurgery, Showa University Koto Toyosu Hospital, Tokyo, Japan

Received: March 10, 2021; Accepted: September 1, 2021 Corresponding author: Sadayoshi Nakayama. Department of Neurosurgery, Showa University Fujigaoka Hospital, 1-30, Fujigaoka, Aoba-ku, Yokohama, Kanagawa 227-8501, Japan

Email: sadanakayama@hotmail.co.jp

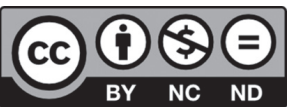

This work is licensed under a Creative Commons Attribution-NonCommercialNoDerivatives International License.

(C)2021 The Japanese Society for Neuroendovascular Therapy nerves must be considered, but transarterial balloonassisted embolization facilitated liquid embolic substance control in several studies, preventing complications. ${ }^{3-5)}$ In this study, we report a patient in whom transarterial balloonassisted embolization for pterygopalatine fossa arteriovenous malformation (AVM) related to venous congestion of the superior ophthalmic vein (SOV), which is rare, was performed without complications.

\section{Case Presentation}

A 57-year-old man presented with congestion of the right bulbar conjunctiva. He consulted a local clinic of ophthalmology. The administration of an eye drop preparation did not relieve the symptom and MRI was performed at his previous hospital. Under a tentative diagnosis of a carotidcavernous fistula, he was referred to our hospital. There was no contributory, no head or facial trauma, or otorhinolaryngological or ophthalmological surgery before his presentation. He presented with congestion of the right bulbar conjunctiva, right exophthalmos, right palpebral edema, and mild right ocular motility disorder in all directions (Fig. 1A). There was no other type of cranial neuropathy, including trigeminal neuropathy, which is characterized by pain and facial dysesthesia. The left and right ocular 

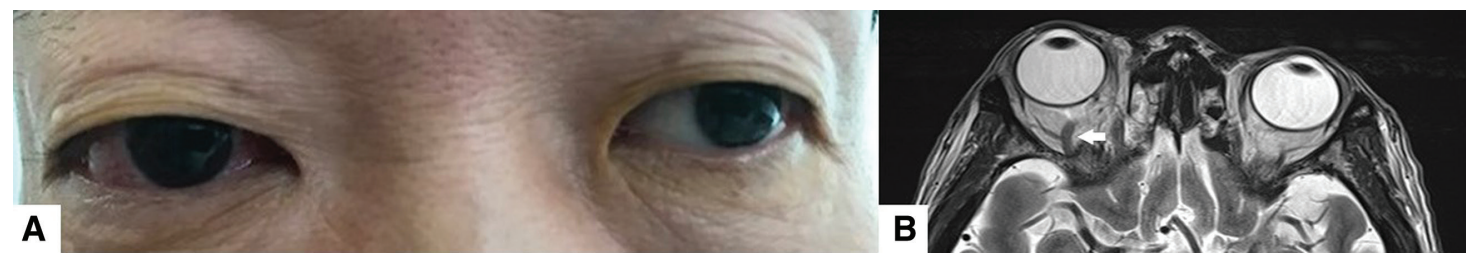

Fig. 1 (A) On the initial consultation, congestion of the right bulbar conjunctiva, exophthalmos, and right palpebral edema were observed. (B) T2-weighted MRI on the initial consultation revealed dilation of the right SOV (arrow) and right exophthalmos. SOV: superior ophthalmic vein

pressures were 10 and $22 \mathrm{mmHg}$, respectively. MRI-T2weighted imaging (T2WI) revealed dilation of the right SOV and right exophthalmos. Contrast-enhanced MRI-T1weighted imaging (T1WI) demonstrated abnormal blood vessels aggregating in the pterygopalatine fossa (Fig. 1B). On cerebral angiography, aggregated blood vessels of the pterygopalatine fossa in which a muscular branch from the first/second segment junction of the $\mathrm{OA}$ to the inferior medial posterior area and another muscular branch from the second/third segment junction to the medial posterior area flowed from the superior area, and a maxillary artery branch, artery of the superior orbital fissure (ASOF), ${ }^{6}$ from the inferior area were observed (Figs. 2A, 2B, 2D, 2E, and 3A-3C). The primary outflow tract was from the inferior ophthalmic vein (IOV) to the anterior superior temporal vein- or angular vein-mediated facial vein via the SOV. Severe stenosis of an outflow tract to the posterior cavernous sinus via the SOV was observed, exhibiting slight outflow (Fig. 2C and 2F). There were superior and inferior inflow sites for aggregated blood vessels of the pterygopalatine fossa, and a diagnosis of arteriovenous shunt disease at this site was made. However, it was difficult to differentiate AVM from an arteriovenous fistula (AVF) (Fig. 3D). A venous approach was considered difficult for the following reasons: the SOV to angular vein was markedly tortuous, the confluence angle between the SOV and IOV was sharp, and torsion was marked even at the shunt site in the IOV; therefore, TAE was selected. A 7-Fr FUBUKI guiding catheter (Asahi Intecc, Aichi, Japan) was inserted into the right external carotid artery. A4-Fr diagnostic catheter was inserted into the right internal carotid artery. A 4.2-Fr FUBUKI (Asahi Intecc) was guided into the right maxillary artery through the 7-Fr FUBUKI guiding catheter. A Scepter C $4 \times 11 \mathrm{~mm}$ (MicroVention) was guided to an area immediately before the ASOF and aggregated blood vessels. The blood vessel was occluded with the Scepter C and ONYX 18 infusion was started. When the total infusion volume reached $0.31 \mathrm{~mL}$, ONYX inflow to the IOV was observed. Both internal and external carotid arteriography confirmed the disappearance of the arteriovenous shunt
(Fig. 3E-3I). During ONYX infusion, attention was paid to prevent inflow to a branch feeder of the OA or peripheral collateral pathway. All symptoms reduced a few days after surgery. The patient was discharged 5 days after surgery. During the 29-month postoperative follow-up period, there was no recurrent symptom or recurrence on MRI.

\section{Discussion}

In the present case, a shunt was present in the pterygopalatine fossa inferior to the pterygopalatine fossa-inferior orbital fissure junction. To our knowledge, no study has reported arteriovenous shunt disease of the pterygopalatine fossa. As feeders, an OA branch was responsible for inflow from the superior area, and a maxillary artery branch, ASOF, for inflow from the inferior area at distant sites, respectively. Considering the possibility of AVM, a therapeutic strategy was selected. Aggregated blood vessels were present in the pterygopalatine fossa, exhibiting outflow to the IOV through the inferior orbital fissure. The pterygopalatine fossa involves the courses of the second and third branches of the trigeminal nerve, pterygopalatine ganglions, and sphenopalatine and Vidian arteries as maxillary artery branches, and a large number of nerves and arteries, including ASOF; therefore, direct surgery is invasive and difficult. ${ }^{7)}$ Furthermore, in the present case, symptom exacerbation was noted and radiotherapy requiring a specific time was not selected; endovascular treatment was adopted. Arteriovenous shunt diseases include AVM and AVF, but it is difficult to differentiate a micronidus AVM from an AVF, as demonstrated in the present case. For endovascular treatment, AVF can be treated by embolization of a drainer. However, in AVM patients, embolization of a drainer alone may induce hemorrhage. In the present case, when selecting a therapeutic strategy, approaching was difficult and transvenous embolization (TVE) was not selected considering the AVM-related risk. TAE through the ASOF, which facilitated catheter insertion to the periphery of a feeder adjacent to the nidus, was instead performed to enable treatment even in the presence of an AVF. Several 

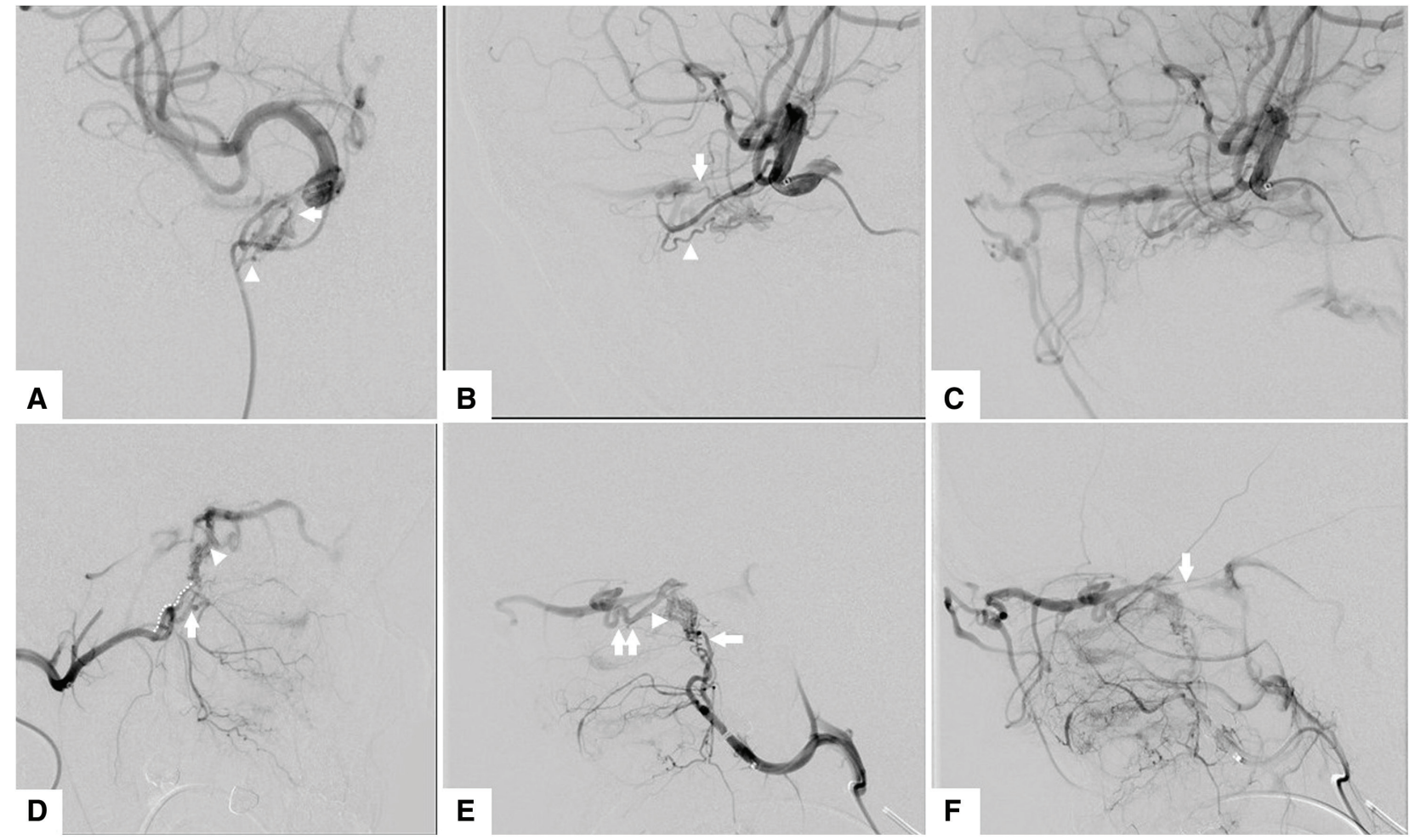

Fig. 2 (A) Arterial phase of a frontal view on right internal carotid arteriography before treatment. (B) Arterial phase of a lateral view on right internal carotid arteriography before treatment. A portion of the superior area of the shunt was visualized from a muscular branch (arrowheads in A and B) from the first/second segment junction of the ophthalmic artery to the inferior medial posterior area and another muscular branch (arrows in A and B) from the second/third segment junction to the medial posterior area. (C) Venous phase of a lateral view on right internal carotid arteriography before treatment. The main drainers outflowed to the front are superficial temporal vein and facial vein mediated by the angular vein. (D) Arterial phase at $\mathrm{RAO} 40^{\circ}$ on selective right external carotid arteriography before

studies reported that transarterial balloon-assisted embolization with a dual-lumen balloon catheter for the infusion of a non-adhesive liquid embolic substance, represented by ONYX, reduced the incidence of complications through favorable embolic substance control, improving the radical cure rate. ${ }^{3-5)}$ In the present case, after a Scepter $C$ was guided to the periphery, a feeder was occluded with a balloon and ONYX was infused, facilitating subtle infusion control and embolization of abnormal aggregated blood vessels and drainers without excessive embolization of the feeder and its branch. When infusing ONYX through a dual-lumen balloon catheter, such as Scepter C, there is no difficulty in guiding to the periphery, risk of balloonrelated vascular injury on guiding, or ONYX regurgitation; therefore, the migration of ONYX into other feeders, collateral pathways, or nerve-nourishing blood vessels must be considered. When performing TAE with a liquid embolic substance, including in the present case, we directly mark a treatment. Sphenopalatine and infraorbital arteries branched from the third part of the maxillary artery (arrow). The artery of the superior orbital fissure (dotted line) also functioned as a feeder, exhibiting inflow to the shunt site (arrowhead) via aggregated blood vessels. (E) Arterial phase of a lateral view on selective right external carotid arteriography before treatment. The entire shunt (arrowhead) was visualized from the artery of the superior orbital fissure (arrow). Outflow to the IOV (double arrow) was observed. (F) Venous phase of a lateral view on selective right external carotid arteriography before treatment. Severe stenosis of the outflow tract from the SOV to the cavernous sinus was noted (arrow). IOV: inferior ophthalmic vein; RAO: right anterior oblique; SOV: superior ophthalmic vein

feeder as an index of infusion stoppage or inflow direction on a monitor to prevent embolic-substance migration. To our knowledge, no study has reported arteriovenous shunt disease of the pterygopalatine fossa, but regarding periorbital AVF, for which the hemodynamics and condition often resemble those in the present case, TAE was selected for 27 patients reported by Sato et al. and for 10 of 31 patients for whom treatment methods were able to be confirmed from other case reports. ${ }^{8-12)}$ However, as a complication, visual field defect was observed in three patients. Furthermore, liquid embolic substances may migrate into fine collateral pathways or nerve-nourishing blood vessels that cannot be visualized on angiography, leading to occlusion. Therefore, when infusing a liquid embolic substance through the third part of the maxillary artery, involving the ASOF, as demonstrated in the present case, the possibility of visual field defect, ocular motility disorder, facial dysesthesia, facial neuropathy, or masticatory disorder must be sufficiently explained 

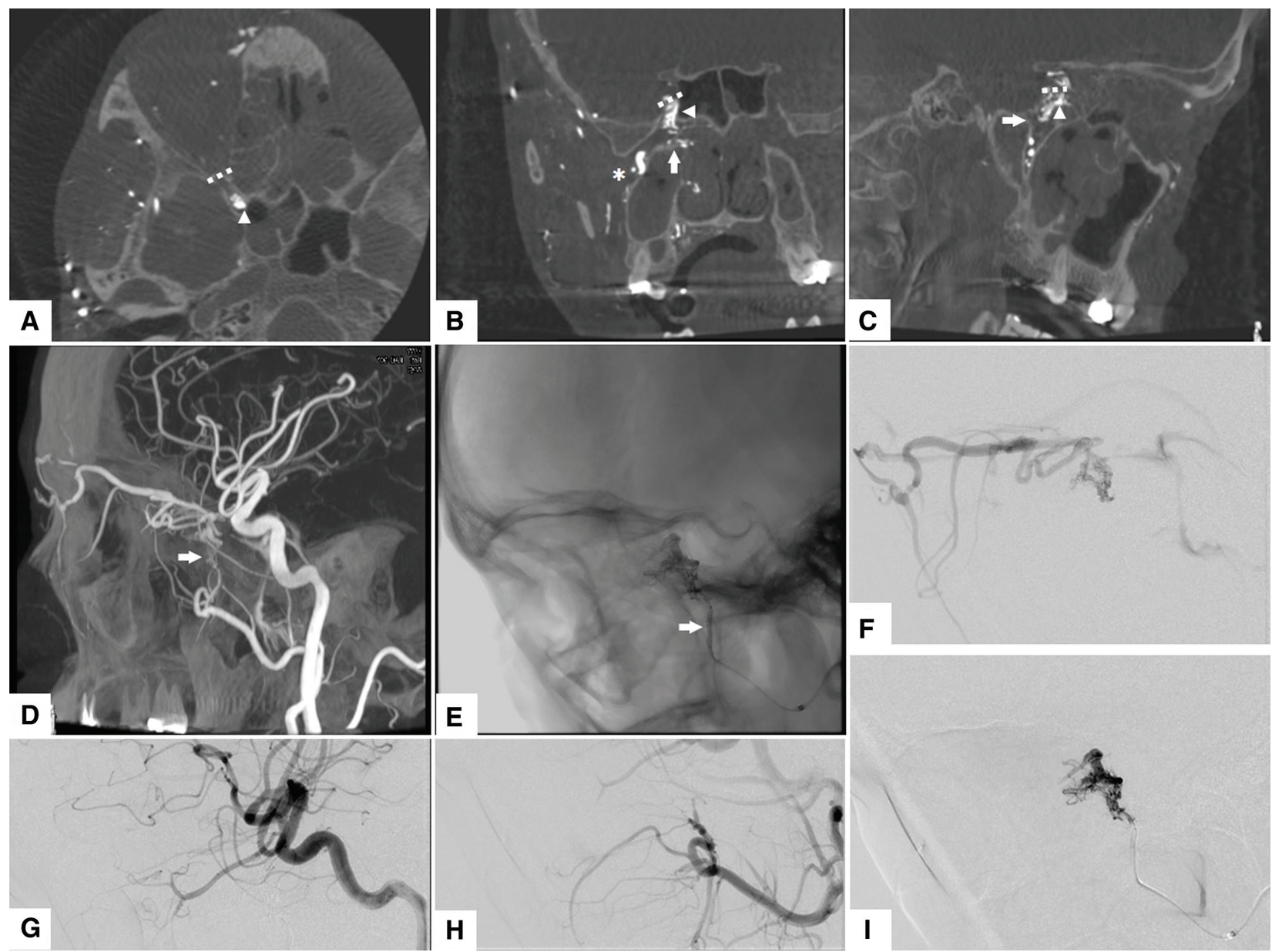

Fig. 3 (A) Axial section on external carotid arteriography (thin slab maximum intensity projection, $0.4-\mathrm{mm}$ thick). In an area posterior to the inferior orbital fissure (dotted line), a shunt (arrowhead) was observed. (B) Coronal section on external carotid arteriography (thin slab maximum intensity projection, $0.4-\mathrm{mm}$ thick). An inferior orbital fissure (dotted line), sphenopalatine cleft (arrow), and pterygomaxillary fissure (asterisk) were observed. In an area medial to the pterygopalatine fossa inferior to the inferior orbital fissure, a shunt (arrowhead) was noted. (C) Sagittal section on external carotid arteriography (thin slab maximum intensity projection, $0.4-\mathrm{mm}$ thick). A shunt (arrowhead) was observed between the inferior orbital fissure (dotted line) and foramen rotundum (arrow). (D) Sagittal section on

before surgery because anastomosis with the $\mathrm{OA}$, inferior lateral trunk, or meningohypophyseal trunk is present, and the pterygopalatine fossa involves the courses of the pterygopalatine ganglions and trigeminal nerve. A previous study reported that coil embolization of other feeders before the infusion of a liquid embolic substance prevented its migration, ${ }^{9}$ ) suggesting the usefulness of coil embolization of other feeders. When adopting this method, an approach route for additional treatment may be occluded; thus, feeders to be blocked with coils must be sufficiently reviewed.

\section{Conclusion}

We reported a patient with pterygopalatine fossa AVM related to hypoperfusion of the SOV, which may be rare. Transarterial balloon-assisted embolization may improve the radical cure rate and safety of treatment.

\section{Disclosure Statement}

The authors declare no conflicts of interest. 


\section{References}

1) Rezende MT, Piotin M, Mounayer C, et al. Dural arteriovenous fistula of the lesser sphenoid wing region treated with Onyx: technical note. Neuroradiology 2006; 48: 130-134.

2) Abud TG, Nguyen A, Saint-Maurice JP, et al. The use of Onyx in different types of intracranial dural arteriovenous fistula. AJNR Am J Neuroradiol 2011; 32: 2185-2191.

3) Clarençon F, Di Maria F, Gabrieli J, et al. Double-lumen balloon for Onyx ${ }^{\circledR}$ embolization via extracranial arteries in transverse sigmoid dural arteriovenous fistulas: initial experience. Acta Neurochir (Wien) 2016; 158: 1917-1923.

4) Kim JW, Kim BM, Park KY, et al. Onyx embolization for isolated type dural arteriovenous fistula using a dual-lumen balloon catheter. Neurosurgery 2016; 78: 627-636.

5) Zamponi JO, Trivelato FP, Rezende MTS, et al. Transarterial treatment of cranial dural arteriovenous fistulas: the role of transarterial and transvenous balloon-assisted embolization. AJNR Am J Neuroradiol 2020; 41: 2100-2106.

6) Kiyosue H, Tanoue S, Hongo N, et al. Artery of the superior orbital fissure: an undescribed branch from the pterygopalatine segment of the maxillary artery to the orbital apex connecting with the anteromedial branch of the inferolateral trunk. AJNR Am J Neuroradiol 2015; 36: 1741-1747.
7) Hitotsumatsu T, Rhoton AL. Unilateral upper and lower subtotal maxillectomy approaches to the cranial base: microsurgical anatomy. Neurosurg 2000; 46: 1416-1452.

8) Sato H, Miyachi S, Hashimoto A, et al. Transarterial embolization of intraorbital dural arteriovenous fistula: a case report. No Shinkei Geka 2019; 47: 777-783.

9) Terakado T, Nakai Y, Tsukada K, et al. A case of orbital dural arteriovenous fistula with exophthalmos treated by transarterial embolization. JNET J Neuroendovasc Ther 2019; 13: 314-319.

10) Lv X, Li W, Liu A, et al. Endovascular treatment evolution for pure intraorbital arteriovenous fistula: three case reports and literature review. Neuroradiol J 2017; 30: 151-159.

11) Akamatsu $Y$, Kubo $Y$, Chida K, et al. Intraorbital arteriovenous fistula presenting with impaired extraocular movement after a provocation test at the third segment of the ophthalmic artery. World Neurosurg 2019; 131: 1-5.

12) Diaz R, Rodriguez R, Almeida P, et al. Spontaneous intraorbital arteriovenous fistula: a rare cause of proptosis. Cureus 2019; 11: e5984. 